\title{
INFORME DE CASOS:
}

\section{REPORTE DE INSUFICIENCIA RENAL PRODUCIDA POR Leptospira Interrogans SEROVAR Pomona EN COLOMBIA (1)}

\author{
FORTUNATO OSPINO*, LIGIA SEDANO de DE LEON**, MANUEL ISAAC GALLEGO**
}

Se describe la presentación de un caso de leptospirosis producida por $L$. pomona en un paciente de 20 años de edad, trabajadora habitual en un molino de arroz y caracterizado por un síndrome de insuficiencia renal y hepática

El cuadro clínico consistió principalmente en malestar general, anorexia, vómito, fiebre, diarrea, ictericia y hemorragias subconjuntivales.

Los exámenes de laboratorio mostraron alteraciones, en los niveles de nitrógeno ureico, creatinina, depuración de creatinina, concentración de cloro, fosfatasa alcalina y bilirrubina directa lo cual podría indicar un daño en el funcionamiento hepático y renal.

Las alteraciones en la velocidad de sedimentación, hemoglobina, hematocrito y leucocitos (Tabla 1) explican la anemia e infección ocasionada por la $L$. pomona en la paciente.

El diagnóstico de leptospirosis fue confirmado por el aislamiento del microorganismo de la orina a pesar de no haber detectado anticuerpos en el suero de la paciente.

Se pone en evidencia la necesidad de realizar siempre el diagnóstico diferencial con leptospirosis en todo síndrome hepatorrenal, especialmente en los casos con antecedentes epidemiológicos o que por la sintomatología clínica se sospeche la infección.

Palabras claves adicionales: zoonosis, leptospirosis.

\section{INTRODUCCION}

La leptospirosis es una zoonosis raramente diagnosticada en seres humanos, no obstante ser considerada una enfermedad frecuente en todas las especies de animales domésticos en el país, no se le ha dado la importancia que merece como zoonosis en la salud humana, por no haberse valorado su incidencia debido a la falta de divulgación de su importancia y de las técnicas corrientes de diagnóstico bacteriológico y serológico.

Por las razones anteriores se presenta este trabajo con el fin de interesar a los profesionales de la salud sobre la enfermedad, sintomatología y la importancia de su diagnóstico.

(1) Contribución del Programa de Microbiología del ICA y del Departamento de Clínicas Médicas, Unidad de Infectología de la UPI 03 Clínica San Pedro Claver.

* Médico Infectólogo UPI 03 San Pedro Claver y Profesor Asistente del Departamento de Microbiología y Parasitología, Facultad de Medicina, Universidad Nacional de Colombia. A.A. 57246.

* * Respectivamente: Bacterióloga y Médico Veterinario, Programa de Microbiología, Laboratorio Investigaciones Médicas Veterinarias. ICA, AA. 29743, Bogotá. 


\section{REVISION DE LA LITERATURA}

La leptospirosis ha sido considerada como una zoonosis en la cual juega un papel importante en los mecanismos de transmisión de la entidad, los roedores, los animales silvestres y las diferentes especies domésticas como bovinos y caninos.

La enfermedad produce en los seres humanos manifestaciones clínicas que varían ampliamente. Puede presentarse como una patología severa febril, meníngea o ictérica o transcurrir con sintomatología leve, sin ofrecer un cuadro clínico preciso. La incidencia en la presentación de los síntomas depende de la frecuencia con que sean diagnosticadas las infecciones leves y como este tipo de infección no se diagnostica corrientemente, la incidencia relativa de síntomas severos puede parecer más alta que lo que realmente es (12-15).

$\mathrm{La}$ forma febril o anictérica está frecuentemente acompañada de fiebre, cefalea y dolores generalizados; este cuadro es tan inespecífico que el diagnóstico de la leptospirosis no se sospecha y la condición es interpretada como influenza o una "virosis".

La forma febril con falla hepatorrenal se presenta como consecuencia del agravamiento de la forma benigna. Esta presentación está acompañada de vómito, ictericia y síntomas meníngeos que pueden estar enmarcadados por signología hepática y renal. Cuando la enfermedad es severa el paciente puede sucumbir al final de la segunda semana como consecuencia de la falla hepática y renal, con aparición de hemorragias y estado de coma (14-15).

Según la Organización Mundial de la Salud (15) la enfermedad constituye un riesgo ocupacional para los trabajadores de los arrozales, de los ingenios de caña de azúcar, campesinos, trabajadores del alcantarillado, mineros, médicos veterinarios, personas que crían animales, empleados de mataderos, manejadores de pescado y personal militar. De igual manera, se registran brotes entre personas expuestas al agua dulce y contaminada por la orina de animales domésticos o salvajes, o la orina o tejidos de animales infectados. También constituye un peligro para los bañistas, deportistas y excursionistas en zonas afectadas.

Como reservorios con frecuencia figuran los animales de granja domésticos, las ratas y otros roedores (7-9-11).
El modo de transmisión se realiza por contacto de la piel con agua, suelo húmedo o vegetación contaminadas con la orina de animales infectados, lo cual es favorecido si se presentan escoraciones (12-14-15).

Según Szyfres (14) en los países desarrollados su impacto en la salud tiende a disminuir, gracias a la mecanización de tareas rurales, a las mejores condiciones medioambientales y a la generalización de las medidas preventivas contra la infección en los animales domésticos. En cambio en los países en desarrollo, la situación es bien distinta, ya que el $50 \%$ de la población vive en zonas rurales en estrecho contacto con toda clase de animales y expuestos a un alto riesgo de infección.

En América del Sur se han aislado diferentes serotipos de Leptospira: $L$. icterohaemorrhagiae, $L$. canicola y $L$. grippotyphosa, $L$. andamana, $L$. pyrogenos, $L$. hebdomadis y $L$. pomona, principalmente en bovinos, porcinos y caninos.

En Colombia, sólo se ha informado la presentación en seres humanos de $L$. canicola y existen algunos estudios serológicos esporádicos realizados especialmente en trabajadores de mataderos, quienes han dado una positividad principalmente a $L$. pomona y $L$. autumnalis (5-10). En Medellín, Bravo y Restrepo (1) identificaron serológicamente $L$. canicola en un paciente masculino, aseador de corrales en una plaza de ferias y quien antes de desarrollar los síntomas estuvo bañándose en una quebrada de aguas negras. Este paciente presentó entre otros síntomas ictericia, hepatomegalia y meningitis con cefalia, trismus y rigidez de nuca, además de alteraciones renales. El cuadro clínico descrito por Bravo y Restrepo (1) corresponde al que se observa en las formas graves de la enfermedad de Weil. Esta fue hasta el momento la primera comprobación clínica y serológica de la leptospirosis humana en Colombia.

De acuerdo con cifras registradas en el país, basadas principalmente en encuestas serológicas, existen niveles altos de reactores en los animales domésticos. Estas investigaciones han revelado procentajes que fluctúan entre el $14.7 \%$ y el $62.6 \%$ (14), y en encuestas mas recientes se han detectado porcentajes que oscilan entre 14 y $21 \%$ de reactores serológicos en ganado de leche de diferentes áreas geográficas del país. 
Con base en las cifras anteriores se puede pensar en la posibilidad de un porcentaje alto de animales domésticos infectados, los cuales podrían ser una fuente de contagio muy importante en humanos y en consecuencia es de esperar una frecuencia mayor de casos clínicos en Colombia.

El presente trabajo tiene como fin presentar un caso de leptospirosis humana en una paciente procedente de Villavicencio, así como el aislamiento del agente etiológico responsable.

\section{MATERIALES Y METODOS}

\section{Descripción del paciente.}

Paciente de 20 años, estado civil soltera, natural y procedente de Villavicencio, trabajadora de un molino de arroz de la ciudad citada. Relata que el día 31 de enero de 1986 presentó los primeros síntomas de la enfermedad, caracterizados por malestar general, anorexia, vómito postprandial, astenia, fiebre, diarrea, orinas oscuras y turbias. La paciente fue hospitalizada en Villavicencio el 7 de febrero de 1986; posteriormente fue remitida a la CPI-03 San Pedro Claver, ISS. de Bogotá.

\section{Exámenes de laboratorio.}

En la Tabla 1 se encuentran consignados los exámenes de laboratorio practicados al momento de su ingreso al hospital: química sanguínea: nitrógeno uréico, concentración de creatinina, bilirrubina total y bilirrubina directa, concetración de sodio, potasio y cloro en sangre.

Hematología: hemoglobina, hematocrito, leucocitos, neutrofilos, linfocitos, plaquetas, tiempo de protrombina y velocidad de sedimentación de los eritrocitos. Parcial de orina: densidad, presencia de leucocitos y bacterias.

TABLA I. RESULTAdos DE LABORATORIO CLIMICO EN UMA PACIENTE AFECTAOA POR LEPTOSPIROSIS L. POMONO

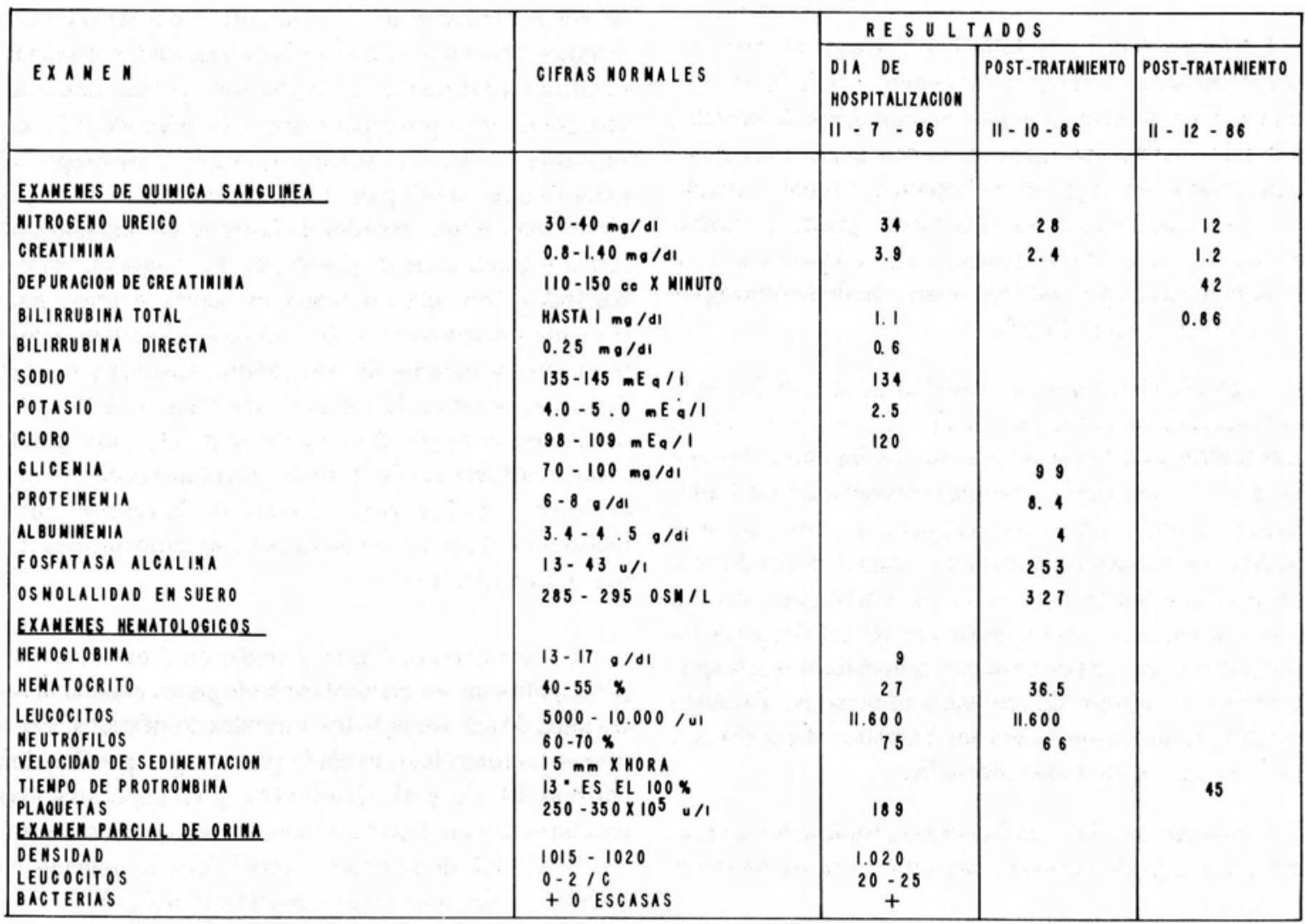


El 10 y 12 de febrero se le practicaron nuevas pruebas de laboratorio para observar la evolución del proceso, sin embargo, los tipos de exámenes no coinciden debido a que la paciente fue trasladada de Villavicencio a Bogotá bajo el cuidado de personal médico diferente.

Al Programa de Microbiología del laboratorio de Investigaciones Médicas Veterinarias (LIMV) se enviaron el $10 \mathrm{de}$ febrero muestras de orina para practicar cultivo de leptospira y muestras de sangre para realizar pruebas de microaglutinación para leptospirosis.

La muestra de orina fue sembrada inmediatamente llegó al laboratorio en medio Ellinghausen semisólido y medio Fletcher, mediante el sistema de diluciones. Los cultivos se mantuvieron en incubación a $28^{\circ} \mathrm{C}$ y observación durante 25 días al cabo de los cuales se detectó la presencia de Leptospiras. Estas se repicaron en medio Ellinghausen semisólido y posteriormente se enviaron al Centro Panamericano de Zoonosis para identificación del serovar.

La muestra de suero sanguíneo fue sometida a la prueba de microaglutinación empleando como antígenos los siguientes serovars de Leptospira interrogans; $L$. pomona, $L$. canicola, $L$. icterohemorrhagiae, $L$. hardjo, $L$. grippotyphosa, $L$. wolffi, $L$. australis, $L$. bataviae, $L$. celledoni, $L$. ballum, $L$. tarassovi, $L$. andamana y L. pyrogenos.

Las técnicas de cultivo y serología se hicieron de acuerdo con las normas del Centro Panamericano de Zoonosis (2).

Una vez realizado el diagnóstico de leptospirosis se comenzó el tratamiento de la paciente a base de dextrosa al $5 \%$ en agua destilada, penicilina cristalina, gentamicina y dieta líquida.

\section{RESULTADOS}

\section{Examen Clínico}

Antecedentes personales: Menarquia a los 18 años, tratamiento con endocrinólogo, ciclos 30 x 3 y última mestruación enero 11 de 1986 . Hace año y medio le practicaron la paroscopia, la cual reveló ovarios poliquísticos.
Al examen clínico se observó desarrollo corporal normal, regulares condiciones generales orientada en tiempo y espacio: T A 100 x $60, \mathrm{~F}$ c 86 por minuto y temperatura axilar de $38^{\circ} \mathrm{C}$.

Conjuntivas oculares y piel ictéricas, hemorragias subconjuntivales, lengua saburral y húmeda, a la auscultación cardiopulmonar se escucharon escasos estertores subcrepitantes basales, abdómen blando sin visceromegalias, reflejos osteotendinosos y sensibilidad normales.

A partir del tercer día de hospitalizada hubo defervescencia y en los días subsiguientes el vómito, las nauseas y la diarrea, lo mismo que la ictericia, cefalea y las hemorragias cojuntivales fueron cediendo hasta volverse negativos.

\section{Patología Clínica}

En la Tabla 1 figuran los resultados de laboratorio practicados al momento del ingreso al hospital y los controles realizados a los 3-5 días después del tratamiento.

\section{Urocultivo}

El cultivo para leptospiras a partir de la orina del paciente fue positivo para $L$. interrogans serovar pomona, clasificación realizada por el Centro Panamericano de Zoonosis. (Oficina Sanitaria Panamericana en Bogotá Ref. Col./ SP4/ 204-86).

\section{Prueba de Microaglutinación}

La prueba de microaglutinación dió resultados negativos para todos los serotipos empleados inclusive $L$. pomona.

\section{DISCUSION}

En primer lugar hay que tener en cuenta que la enfermedad se presentó en una persona con un riesgo de infección bastante alto, por ser empleada de un molino de arroz. Este cultivo requiere condiciones de humedad y temperatura óptimas muy favorables para el mantenimiento de las leptospiras. En este tipo de cultivo los animales silvestres, como las ratas, depositan la orina con una gran cantidad de leptospiras y aunque el medio ambiente puede no ser favorable para 
la conservación del microorganismo, la contaminación al ser humano puede presentarse al entrar en contacto con un gran número de microorganismos en un momento determinado, los cuales posteriormente mueren en un tiempo relativamente corto. Por otra parte, la cantidad de gérmenes necesarios para producir una infección es relativamente pequeña (9) sobre todo si la cepa es lo suficientemente virulenta y específica de especie (7).

La enfermedad se manifiesta en dos formas: una leve caracterizada por trastornos generales, los cuales pueden ser confundidos con una enfermedad febril pasajera; la otra forma, menos frecuente, se caracteriza por compromiso hepatorrenal, como el descrito en este trabajo con la presentación de toda la sintomatología propia de la enfermedad en seres humanos, como son: vómito, ictericia, hemorragias, fiebre y anorexia principalmente (3-12-13-15).

Los hallazgos de laboratorio guardan relación con los descritos para la enfermedad (3-8-12-15). En la Tabla 1 puede observarse que las cifras anormales de los diferentes análisis presentaron una tendencia de retorno a cifras normales después del tratamiento de la infección.

Dentro de los parámetros de funcionamiento renal, se encontró alteración inicial en los niveles de creatinina y de concentración de cloro, los cuales fueron altos al ser hospitalizada la paciente. La concentración de creatinína retornó a niveles normales en el tercer examen realizacio.

Las demás pruebas de funcionamiento renal estuvieron dentro de los límites normales.

La alteración en los niveles de creatinina indican una deficiencia en la filtración glomerular confirmada por los bajos niveles de depuración observados en el tercer examen. La concentración alta de cloro indica aumento en la reabsorción o falla en la eliminación de este elemento a nivel de los tubulos renales.

Para explicar los niveles bajos de nitrógeno uréico detectados el día 5o. post-tratamiento es necesario pensar en la posibilidad de una dieta protéica baja, a pesar de que las concentraciones de proteína fueron normales.
En las pruebas de funcionamiento hepático se observa alteración en las cifras de fosfatasa alcalina y bilirrubina directa. Estas alteraciones indican incapacidad del hígado para eliminar la bilirrubina conjugada lo cual produce una icteria post-hepática y permite el aumento de la fosfatasa alcalina.

Los exámenes hematológicos indican una aparente anemia e infección, las cuales están correlacionadas con la presencia de $L$. pomona gérmen que produce hemólisis por destrucción de la membrana de los glóbulos rojos.

Los exámenes serológicos dieron resultados negativos tanto para el serovarL. pomona como para los otros antígenos empleados en el diagnóstico. En la literatura médica no se encontraron referencias sobre los casos humanos en las cuales se mencione la disparidad entre la respuesta serológica y el aislamiento de leptospira. Es oportuno mencionar que en bovinos se encuentran animales eliminando los microorganismos con serología negativa y animales con serología positiva y aislamiento negativo (4), por lo tanto es posible que en este caso se haya presentado un fenómeno similar.

\section{CONCLUSIONES}

Con este trabajo queda demostrada mediante aislamiesto la presencia de Leptospira interrogans serovar pomona como agente etiológico de un diagnóstico de insuficiencia renal y síndrome ictérico en una paciente procedente de Villavicencio y quien se hallaba bajo condiciones ambientales propicias para desarrollar la infección.

Es necesario tener en cuenta el diagnóstico serológico y bacteriológico de leptospirosis en todos aquellos casos que presenten un cuadro clínico semejante, o que por los antecedentes epidemiológicos ofrezcan la posibilidad de una infección por este organismo.

\section{SUMMARY}

It is described a leptospirosis case produced by Leptospira pomona in a 20 years old woman working in a rice mill, and characterized by a sindrome of hepatic and renal insufficiency. 
The disease was manifested by discomfort, anorexia, vomiting, fever, diarrhea, jaundice and subconjuntival bleeding.

Clinical tests shawed disturbances in the levels of Creatinine, blood chlorine, alkaline phosphatase and direct bilirrubin indicating a renal and hepatic dysfunction.

Hematic disturbances in erythrocyte sedimentation rate, hemoglobin, haematocrit and leukocytosis showed the infection and anemia produced by $L$. pomona in the patient.

It is called the attetion to make always a differential diagnosis in all suspicious cases of leptospirosis specially when the epidemiological background and symtoms make suspect a disease produced by Leptospira species.

\section{BIBLIOGRAFIA}

1. Bravo C, Restrepo M. Leptospirosis Humana en Colombia. Antioquia Médica 1969; 19: 947.

2. Centro Panamericano de Zoonosis. Leptospirosis. Manual de métodos para el diagnóstico de laboratorio. Nota Técnica No. 30. 1985; p 46.

3. Edwars CN, Nichols GD, Hassell TA, Evarard COR, and Calleder J. Thrombocytopenia in leptospirosis: The absence of evidence for diseminated intravascular coagulation. Am J Trop Med Hyg. 1986; 35: 352.

4. Ellis WA. Recent development in bovine leptospirosis. The Veterinary Annual. 23rd Issued. GSG Grunsel. WEG Hill London 1983; p 91.
.5 García CC. Leptospirosis y Brucelosis en el personal de Matadero de Manizales. Universidad de Caldas. Veterinaria y Zootecnia. 1966; 7: 67.

6. Griffiths IA, Gallego MI, Villamil LC. Factores de infertilidad y pérdidas económicas en ganado de leche. 1982; Publicación ICA 99-2.2-094-82 p 182.

7. Hataway SC, Blackmore DK, Marshall RB. Leptospirosis and the maintenance host: A laboratory mouse model. Research in Veterinary Science 34: 82.

8. Kadis S, Pugh WL. Urea utilization by leptospira. Infection and Immunity. 1974; 10: 783.

9. Michna SW. Leptospirosis The Veterinary Record. 1970; 86: 484.

10. Muñóz RG. Existe Leptospirosis en Colombia. Rev Fac Med Vet Zoot Bogotá, 1957; 21: 590.

11. Myers DM, Cuba CA, Payan MJ. Aislamiento de Leptospira hardjo y otras leptospiras de Armadillos de Argentina. Boletín Oficina Sanitaria Panamericana. 1977; 83: 56.

12. Organización Panamericana de la Salud. El control de las enfermedades transmitidas en el hombre. 1983; Publicación Científica No. 442. Editada por S. Benson, Washington p 486.

13. Ramachandram S, Perea MVF. Cardiac and pulmonary involvement in leptospirosis. Trans Roy Soc Tro Med Hyg. 1977; 71: 56.

14. Zyfres B. Leptospirosis as an animal and Public health problem in Latin America and the Caribbean area. Bull Pan Health Org, 1976; 10: 110.

15. World Health Organization. Guideline for the control of Leptospirosis. 1982; WHO offset publication No. 67 Edited by S. Faine. Geneva p 171. 